\title{
Urban Energy Flux: Spatiotemporal Fluctuations of Building Energy Consumption and Human Mobility-Driven Prediction
}

\author{
Neda Mohammadi, John E. Taylor* \\ School of Civil and Environmental Engineering, Georgia Institute of Technology, Atlanta, GA 30332, USA
}

\begin{abstract}
Urbanization is causing a significant increase in the amount, diversity, and complexity of human activities, all of which have a substantial impact on energy consumption. Current approaches to predicting energy demand at different spatiotemporal levels are functions of the characteristics of either individual buildings or cities and their occupancy levels, or are data-driven, typically taking the form of sensor-based modeling. Nevertheless, accounting for both the spatial and temporal effects of heterogeneous human behavior patterns on buildings' energy use at the city level remains a challenge. In this paper, we examine the temporal manifestation of the fluctuations of energy use in urban buildings driven by spatial mobility patterns of the population. We then present an urban-level spatiotemporal approach for predicting buildings' energy demand. Using a full year of individual positional records from an online social networking platform (Twitter), we introduce a multivariate autoregressive model in reduced principle component analysis space to create monthly predictions of residential building electricity demand generated across 801 spatial divisions that account for $68 \%$ of the electricity used by buildings in the City of Chicago, through a spatial autoregressive model. This model represents an important step forward, incorporating the spatiotemporal energy use fluctuations of urban population activities to create more reliable predictions of demand in future cities.
\end{abstract}

Keywords: Energy Flux; Energy Prediction; Human Mobility; Spatiotemporal Demand; Urban Energy System.

\section{Introduction}

High levels of uncertainty about future energy demands impose limits on the continuing growth of cities worldwide. Urban areas, which have become the most complex built environments in human history, are rapidly expanding in terms of both their size and population [1]. However, subsequent additions to the amount and complexity of daily activities of urban populations may not be able to be sustained without an accompanying substantial increase in their energy infrastructure. Urban areas are now responsible for up to $80 \%$ of the world's total energy consumption $[2,3]$ and the corresponding risks involved in supplying, or failing to supply, sufficient energy resources are inevitable. In particular, urban buildings are determined as the largest energy consuming sector in the economy [4]. Having a clear understanding of the space-time distributions of urban building energy demand [5-7] is thus clearly of paramount importance for both planners and city managers. Models of urban energy demand often tend to focus on quantifiable factors that influence the demand such as technological, socioeconomic, and those related to the infrastructure. Many models neglect the inclusion of human behavior which can be equally impactful [5, 6], and which adds to model uncertainty [9]. Today, with the advancement of technology, quantifying people's activities and behavior is increasingly possible in many ways[10-13]. Human behavior towards energy consumption is relatively well studied at the building level[14,15].

\footnotetext{
* Corresponding author. Tel.: +1-404-894-8021.

E-mail address: jet@gatech.edu.
} 
However, the significance of human behavior on buildings' energy consumption at the urban-level is an important aspect of urban energy demand modeling that is scarcely studied. Despite the profound impact that this aspect brings on spatiotemporal distribution of energy demand and the calls by researchers for incorporating the effects in demand estimations [6, 8], such measures are currently missing from the literature. We need to be able to reliably estimate how much energy will be needed at particular times and locations across a range of urban buildings. Enhancing our ability to understand and manage short and long term urban energy resources is essential if our cities are to continue to thrive and develop into smarter built environments capable of accommodating the growth of technology and the corresponding fluctuating activity patterns of the urban population. Here, we examine the temporal manifestation of the fluctuations of building energy use driven by the spatial mobility patterns exhibited by the city's populations as an indicator for human activities and introduce a spatiotemporal approach for predicting building energy demand in urban areas.

\section{Related Work}

Current approaches to predicting urban building energy consumption at different spatial levels are functions of the characteristics of buildings [17-19], urban information [5,6,20], or rely on sensor-based data-driven approaches [14]. As the effects of rapid increases in complexity and human activities in cities are amplified [21], understanding and managing the end use patterns of energy demand in spatiotemporal dimensions [5], taking into account the uncertainties of human behavior becomes more imperative.

At the city scale, Chaudhary introduced a Bayesian regression model to estimate energy intensity distributions using floor area estimates for various building usages in London [20]. At the neighborhood level, Mikkola and Lund [6] incorporated information on building types, their load profiles, their share of floor area, energy intensity and electricity demand, as well as population density, and city area into their power demand predictive model. At the block (zip code) level, Howard et al.'s [17] energy prediction model is primarily based on building functions in different locations within New York City. Others have proposed spatiotemporal models for building energy demand patterns of power and temperature, taking into account the variability of energy services across residential, commercial, and industrial buildings at the neighborhood and city district levels [5]. In particular, Zhu et al. [7] proposed a time-for-space substitutional model, which evaluates the variations of residential buildings' energy consumption in China driven by seasonality, climate, and socioeconomic factors. Spatiotemporal energy demand models have further been used to examine the potential for renewable energy investments, by capturing the interplay between power demand and renewable sources at multiple spatial scales, taking into account land use and socioeconomic factors [22]. At the building level, these efforts have tended to focus on data-driven methods and sensor-based energy modelling approaches. For example, Jain et al. [23] introduced a sensor-based forecasting model for residential buildings' energy consumption in New York City, using inputs of electricity consumption at different spatiotemporal granularities supplemented with hourly outdoor temperature data. Sensor-based approaches have enabled models of energy consumption to effectively incorporate the behavioral impacts from the human activity patterns at the building level [15]. However, a thorough review of the literature in this area has uncovered no studies exploring the predictability of urban scale energy consumption that incorporate the spatiotemporal effects arising from fluctuations in the activity patterns of the populations at the urban level. In their studies of nationally representative samples, Huebner et al. [24,25] examined the significance of several variables (i.e., building factors, socio-demographics, appliances, and behavior) in residential building energy consumption and found that those variables that are directly driven by people's activities exhibit the strongest predictive power. Urban population activities are a major source of change affecting future 
demand and thus serve as the foundation of our spatiotemporal estimations of energy use towards more reliable predictions. Researchers have not found it easy to study the dynamics of individual activities at different spatial and temporal resolutions because the only data available was from self-reported surveys, monthly bills, and conceptual frameworks were the only measures available to assist effective decision making. Thanks to recent advances in technology, more powerful computers, and the advent of online social networks, urban population data at high spatiotemporal resolutions is now plentiful. The use of humans as sensors has made city-wide human mobility data available [26] through mobile phone signals that incorporate Global Positioning System (GPS) data [11,26,27], smart card commuting data [28], and location-embedded information from online social networks [10,12,29-31], all of which can be applied to provide information on the mobility behaviour of urban populations. The human mobility patterns that are now visible to researchers reveal important information about the way citizens interact with their surroundings as they go about their daily lives.

As individuals move around their urban environment, they drive the energy consumption associated with their location-based activities in different buildings at particular times. The essential challenge facing academic researchers is thus to find a way to anticipate future changes in human activities (human mobility) that will enable them to make reliable predictions of future urban energy demand. A growing body of research has explored the predictability of human mobility [31-35], and new models are continually being proposed that provide more reliable predictions of energy consumption [6,18,23,36,37]. Studies that have focused primarily on predicting human mobility $[33,34,38,39]$ and building human mobility-based predictive models [13] have taken a number of different approaches. Although up to 93\% predictability has been achieved for the movements of individuals [38,39], changes in their predictability can arise due to unforeseen circumstances such as natural disasters [35]. McInerney et al. [33] examined the boundaries of human mobility predictability to anticipate breaks from routine using a Bayesian model, while others analysed the travel patterns of up to 500,000 individuals using Markov chain based models to predict their visited locations, achieving an $88 \%$ theoretical maximum predictability [34], and introduced a mobility model for predicting individuals' locations using point of interest (POI) information [31]. Several studies of human mobility behaviour have adopted a network perspective to predict the future of such networks as well as the proximity of individuals within the networks, showing great precision in anticipating future mobility topology [32]. Predictive models of human mobility have been extensively applied in recent years across applications such as traffic and travel demand predictions [40,41], human activity predictions [13], next place locations [42], epidemics and the spread of viruses [43-45], air pollution [46,47], and transport energy consumption [47,48].

In order to assess the energy use attributable to individuals' urban mobility and employ human mobility as a predictor of future energy consumption, this study focused on the spatiotemporal predictability of energy consumption driven by intra-urban human mobility behavior of the returners population [49] in the City of Chicago. This population has shown to be most representative of the spatial distribution of energy use in urban settings and dominate the spatial dependency between human mobility and energy use compared to their counterpart population (i.e., explorers). We first examined whether the spatial dependencies previously found between human mobility of returners and energy use in urban areas [50] are also manifested in the form of temporal dependencies and whether temporal fluctuations in the returners' mobility still adequately represent and can explain temporal fluctuations in energy consumption. Then, the spatiotemporal predictability of energy consumption gained by examining human interactions with the urban built environment is explored through multivariate autoregressive time series and spatial regression analysis of 8,798,090 positional records collected from an online social networking platform (Twitter), over the course of 12 months (January-December, 2014) and electricity consumption 
across 801 areas in the City of Chicago. Figure 1 shows a five-day cumulative distribution of positional records across the 801 Census Tract boundaries in the city.

Fig. 1. Five-day cumulative distribution of positional records for February 14-18, 2014 over 801 Census Tracts.

\section{Methods}

\subsection{Data}

The datasets used in this study consist of the electricity consumption figures of residential buildings for 801 spatial divisions in Chicago, and 8,798,090 positional records accounting for human mobility within the city. The spatial level used here is an administrative boundary for Chicago, namely the most recent available Census Tract data, which represents an average population of 4000 , and their corresponding electricity consumption measures [51]. The positional record data has been collected from an online social network (Twitter) from individuals in the city of Chicago who have voluntarily publicly shared locationenabled information for their Twitter accounts. Figure 2 shows the distributions of these two variables ((a) residential electricity consumption, and (b) human mobility of the returners population) in the spatial dimension over the course of the month of February, 2014.

\subsection{Radius of Gyration}

In order to quantify the activity patterns of the urban population through human mobility patterns we have selected the radius of gyration $(E q .2)$ as our metric from among the three widely accepted indicators for describing large-scale human mobility patterns: the radius of gyration $r_{g}(t)$, trip distance distribution $p(r)$, and the number of visited locations $S(t)[11,27,34]$.

$$
\begin{aligned}
& r_{c m i}(t)=\frac{1}{N_{(t)}} \sum_{i=1}^{N_{(t)}} r_{i} \\
& r_{g i}(t)=\sqrt{\frac{1}{N_{(t)}} \sum_{i=1}^{N_{(t)}}\left(r_{i}-r_{c m i}\right)^{2}}
\end{aligned}
$$

Here, $N$ is the total number of observations $n$.

The radius of gyration is calculated at two spatial and two temporal levels. First, the individual level $r_{g i}(t)$-which represents the characteristic distance traveled by a user when observed in time $t$-is obtained per Census Tract per individual per day. Second, the Census Tract level $r_{g a}(t)$ is obtained per Census Tract over each time frame (i.e., month-by-month). Every $r_{g \mathrm{a}}(t)$ represents the deviation of the $r_{g \mathrm{i}}(t) s$ from the corresponding center point. This indicator is used to describe the patterns of human mobility across the Census Tracts.

\subsection{Spatial Autocorrelation}

Spatial autocorrelation [52] was used to assess of the extent to which the spatial distribution of the data is compatible with spatial randomness. Spatial autocorrelation tested the spatial independence of human 
mobility and electricity consumption across 801 spatial divisions in the City of Chicago. Moran's $I$ [53] (Eq. 3), which ranges from -1 (most dispersed) to 1 (most clustered), was used to describe the degree of spatial concentration or dispersion for these variables, with large values for $I$ showing clusters of large values that are surrounded by other large values, namely (I+)-spatial clustering, and (I-)-spatial dispersion, indicating large values that are spatially enclosed by smaller values.

$$
I=\frac{N \sum_{\mathrm{i}=1}^{\mathrm{n}} \sum_{\mathrm{j}=1}^{\mathrm{n}} \mathrm{w}_{\mathrm{ij}}\left(\mathrm{x}_{\mathrm{i}}-\overline{\mathrm{x}}\right)\left(\mathrm{x}_{\mathrm{j}}-\overline{\mathrm{x}}\right)}{\left(\sum_{\mathrm{i}=1}^{\mathrm{n}} \sum_{\mathrm{j}=1}^{\mathrm{n}} \mathrm{w}_{\mathrm{ij}}\right) \sum_{\mathrm{i}=1}^{\mathrm{n}}\left(\mathrm{x}_{\mathrm{i}}-\overline{\mathrm{x}}\right)^{2}}
$$

Here, $n$ represents observations on variable $\mathrm{x}$ at location $i, j$ where $\overline{\mathrm{x}}$ is the mean of variable $\mathrm{x}$, and $\mathrm{w}_{i j}$ are the elements of the weight matrix.

\subsection{Multivariate Autoregressive (MAR) Model in Principal Component Analysis (PCA) Space}

Having accounted for spatial dependencies and the clustering distribution for both human mobility and electricity consumption across spatial divisions in a PCA space, we can examine whether a multivariate autoregressive (MAR) model (Eq. 4) [54] (with monthly time points per spatial division in PCA space) can be used to identify meaningful relationships between the two dimensions. MARs are linear multivariate time series models that characterize interregional dependencies across time and account for the historical influence of the variables on each other. Here, the MAR models the vector of next values for electricity consumption as a weighted linear sum one past vector values of electricity consumption measurement and contains information about the process that generated it. The first model order is determined to be appropriate for this case (i.e., MAR (1)).

$$
E_{(t)}=\hat{\omega}+E_{(t-1)} \hat{\beta}+\hat{\varepsilon}_{t}
$$

Where:

$\hat{\omega}$ : Vector of intercept terms.

$E_{(t-1):}$ Electricity consumption at time $t-1$.

$\hat{\beta}$ : Vector of estimated autoregressive coefficients.

$\hat{\varepsilon}$ : Noise covariance matrix.

It is important to consider an appropriate minimum sample size that adequately represents historical data for electricity and human mobility when building this statistical model. In making do with minimal temporal resolution data, our approach will be representative of this limitation.

\subsection{Spatial Autoregressive (SAR) Model}

To model the spatial interdependencies of the datasets across all 801 spatial divisions, we recommend a spatial autoregressive model to implicitly incorporate the spatial dependence of human mobility data into a covariance structure. According to the spatial autocorrelation results described above, due to the 
presence of an intrinsic spatial structure for both electricity consumption and human mobility in the city of Chicago these measures cannot be regarded as being independent of each other. Spatial regression models are used to examine the relationships between variables and their neighbouring values and allow us to examine the impact that one observation has on other proximate observations. Based on the crosssectional spatial data at time $t$ for human mobility and electricity consumption generated by the MAR model in PCA space, a spatial autoregressive model, namely the simultaneous autoregressive (SAR) spatial lag model shown in (Eq. 5) [55], which represents higher global dependency conditions can be employed. At a global scale, the SAR model implies that the state of the electricity consumption per unit of human mobility for each Census Tract in the city of Chicago is influenced by that of its neighbouring Census Tracts. This model can be used to project the predictions from PCA space to cover all 801 spatial divisions using human mobility as a covariate.

$$
E=\rho W E+r_{g a} \beta+\varepsilon
$$

Where:

\author{
E: $\quad$ Electricity consumption at time $t$. \\ $r_{g a}:$ Human mobility at time $t$. \\ $\rho$ : Spatial autoregressive coefficients the term $\rho W$, represents the spatially lagged dependent variable. \\ $\beta$ : Regression coefficient. \\ $\varepsilon$ : Random error term.
}

\title{
4. Results
}

To investigate whether there is a temporal dependency between fluctuations of the returners mobility and energy use, we captured returners' characteristic intra-urban mobility distance across 801 spatial divisions in the City of Chicago using the radius of gyration $r_{g}(t)$. Through spatial autocorrelation statistics based on a simultaneous consideration of feature locations and feature values, we first measured whether there is indeed a spatial correlation between electricity consumption and their mobility variables, and, if so, whether this correlation holds true in the temporal dimension. In order to test for spatial collinearity and possible dependency structure across the 801 spatial divisions for electricity consumption and human mobility, we performed a spatial autocorrelation analysis to measure the correlations in the spatial dimension using Moran's $I$, which is also a test of independence and then went on to examine whether this dependency held true over the temporal dimension for the entire 12 months of the study.

Table 1 shows the results of the global autocorrelation for both electricity consumption and human mobility. Statistically significant $\left(p\right.$-value $<2.2 e^{-16}$ ) positive values for $I$ over the entire 12 months indicate that returners mobility patterns in the City of Chicago follow a clustering distribution as opposed to a dispersed or random distribution. This indicates that values of human mobility or electricity consumption observed in one location depend on the values observed at adjacent locations, and this dependency exists continuously throughout the course of the year. Figures 3-4 depict the Moran's scatterplots for these values.

Fig. 2. Spatial distribution of (a) Residential electricity consumption, and (b) Human mobility of returners in February across 801 Census Tracts, City of Chicago.

Since electricity consumption exhibited a statistically significant spatial dependence over Chicago's 801 census tracts, we tested the global dependency conditions by modelling the spatial interdependencies, 
via an autoregressive model, thus implicitly incorporating the spatial dependence of the human mobility data into the covariance structure as a function of the number of electricity meters per area. Table 2 shows the spatial autoregressive (SAR) model tested for this study to explicitly examine the impact of returners mobility variables on the consumption of electricity. All spatial parameters were found to be statistically significant, as indicated by low $p$-values for all 12 months. This implies interdependencies for returners mobility and electricity consumption in these areas. Smaller AIC values, and relatively smaller $p$-values for the SAR models indicate that such a dependency exists stronger during that month of the year in comparison to others. The statistically significant spatial correlation for returners' mobility and electricity consumption suggests that human mobility can be a useful spatiotemporal predictor for urban energy use. This indicates that the strength of the association between human mobility and electricity consumption over time depends on spatial location, which can further be contextualized.

Table 1. Spatial Autocorrelation (Moran's I): Electricity consumption, City of Chicago.

\begin{tabular}{rcccccc}
\cline { 2 - 6 } & \multicolumn{2}{c}{ Electricity Consumption } & \multicolumn{3}{c}{ Human Mobility } \\
\hline Month & Statistic & $p$-value & Std. & Statistic & $p$-value & Std. \\
\hline January & 0.077890564 & $4.657 \mathrm{e}-06$ & 4.4325 & 0.0326270440 & 0.04505 & 1.6949 \\
\hline February & 0.0697123924 & $4.186 \mathrm{e}-05$ & 3.9335 & 0.0629956317 & 0.0006563 & 3.2132 \\
\hline March & 0.0627234439 & 0.0002214 & 3.5132 & 0.0647914579 & 0.0004794 & 3.3023 \\
\hline April & 0.0823961291 & $3.144 \mathrm{e}-06$ & 4.5165 & 0.0427146003 & 0.01398 & 2.1979 \\
\hline May & 0.0689312473 & $4.84 \mathrm{e}-05$ & 3.8985 & 0.066187299 & 0.0003722 & 3.3727 \\
\hline June & 0.0725018169 & $2.918 \mathrm{e}-05$ & 4.0193 & 0.0588973269 & 0.001316 & 3.0077 \\
\hline July & 0.0605486473 & 0.0003234 & 3.4112 & 0.074600221 & $7.434 \mathrm{e}-05$ & 3.7933 \\
\hline August & 0.0839596795 & $1.807 \mathrm{e}-06$ & 4.6324 & 0.0781581879 & $3.584 \mathrm{e}-0$ & 3.9707 \\
\hline September & 0.063208821 & 0.0002234 & 3.5108 & 0.0350867002 & 0.03465 & 1.8165 \\
\hline October & 0.0760895855 & $1.661 \mathrm{e}-05$ & 4.1502 & 0.0361323441 & 0.03076 & 1.8697 \\
\hline Dovember & 0.0653879581 & 0.0001383 & 3.6363 & 0.0641694623 & 0.0005351 & 3.2714 \\
\hline
\end{tabular}

In an ideal world, we could predict energy use for any time and location, enabling us to prepare in advance to provide sufficient resources to meet the anticipated demand. Spatiotemporal predictions pose a number of data and analysis challenges, however. Here, we introduce a spatiotemporal approach for predicting energy use in urban areas that combines space-time energy use with human mobility data and accounts for the interdependencies of their fluctuations. Spatiotemporal data are oftentimes not sufficiently accessible or cannot feasibly be collected in either the space or time dimension. Notwithstanding this condition, our data is sparse in time (monthly time intervals), but has higher resolution in space (covering a total of 801 spatial divisions within the City of Chicago). On the other hand, our human mobility data contains higher granularity in both dimensions as humans as dynamic sensors can provide data with fine-grained space-time resolution. Moreover, given the presence of spatial structure for energy consumption and/or human mobility, these variables cannot simply be regarded as being independent of spatial location and are thus subject to incorporating their spatial dependencies. In 
order to account for the spatial dependencies across the 801 spatial divisions and balance the space-time dimensions in our predictive models, we next performed a principle component analysis (PCA) [52]. Table 3 summarizes the global PCA analysis, and reveals that the first six (6) components all have eigenvalues greater than or very close to unity, collectively accounting for $98 \%$ of the spatial variations in the data.

Fig. 3. Moran scatterplot by month (2010): Electricity consumption, city of Chicago.

In order to account for the spatial dependencies over the time and balance the space-time dimensions of our dataset, we then generated autoregressive models in space (SAR) and time (MAR) to predict electricity consumption based on previous spatiotemporal electricity use data, with returners human mobility as a covariate. Using returners mobility across different parts of Chicago as a proxy indicator of electricity consumption behaviour we project estimations of electricity use from a PCA space to spatial scales at a higher resolution than was previously possible. This allows for more reliable spatial predictions for electricity consumption across the temporal dimension. Table 3 extends the statistical results for the multivariate autoregressive (MAR) model in PCA space.

Table 2. Spatial Regression: Electricity consumption per returners' mobility, City of Chicago.

\begin{tabular}{rcccc}
\multicolumn{5}{c}{ Spatial Lag Model (SAR) } \\
\hline Month & Statistic & $p$-value & AIC & AIC for Linear Model \\
\hline March & 0.80157 & $<2.22 \mathrm{e}-16^{* * *}$ & -2890.1 & -2255.2 \\
\hline January & 0.79795 & $<2.22 \mathrm{e}-16^{* * *}$ & -2885.6 & -2273.3 \\
\hline May & 0.80029 & $<2.22 \mathrm{e}-16^{* * *}$ & -2884.2 & -2256.9 \\
\hline February & 0.79995 & $<2.22 \mathrm{e}-16^{* * *}$ & -2875.6 & -2250.1 \\
\hline April & 0.801 & $<2.22 \mathrm{e}-16^{* * *}$ & -2874.8 & -2243.4 \\
\hline August & 0.79298 & $<2.22 \mathrm{e}-16^{* * *}$ & -2862.6 & -2250.7 \\
\hline October & 0.78998 & $<2.22 \mathrm{e}-16^{* * *}$ & -2862 & -2260 \\
\hline June & 0.79497 & $<2.22 \mathrm{e}-16^{* * *}$ & -2848.7 & -2237.5 \\
\hline November & 0.79187 & $<2.22 \mathrm{e}-16^{* * *}$ & -2847.5 & -2243.9 \\
\hline July & 0.77851 & $<2.22 \mathrm{e}-16^{* * *}$ & -2789.4 & -2220.0 \\
\hline December & 0.78378 & $<2.22 \mathrm{e}-16^{* * *}$ & -2787.2 & -2215.5 \\
\hline September & 0.79064 & $<2.22 \mathrm{e}-16^{* * *}$ & -2233.7 & -2842.6 \\
\hline$p<0.05^{*} ; p<0.001 * * ;<0.0001 * * * ;$ & & \\
\hline & & &
\end{tabular}

Table 3. Principal Component Analysis (PCA).

\begin{tabular}{llll} 
& SD & Proportion of Variance & Cumulative Proportion \\
\hline PC 1 & 2.4446039 & 0.4986314 & 0.4986314 \\
PC 2 & 2.2122267 & 0.40834 & 0.9069714 \\
PC 3 & 0.5479213 & 0.0250495 & 0.9320209
\end{tabular}


Table 4 presents the statistical parameter estimations of the proposed SAR model. As indicated by the $p$-value lower than 0.0001 , the model is a statistically significantly representation that can be used to predict electricity consumption per human mobility of the population across spatial divisions. It is important to consider an appropriate minimum sample size that adequately represents historical data for both electricity and human mobility when building such a statistical model. Given the minimal temporal resolution of the data available for this study, our results are representative of this limitation.

Table 4. Electricity consumption per human mobility: (a) Multivariate Autoregressive (MAR) Model; (b) Simultaneous Autoregressive (SAR) Model.

\begin{tabular}{llll} 
MAR & \multicolumn{3}{l}{ SAR } \\
\hline AIC for Simple Linear Model & 2250.6 & AIC for Simple Linear Model & 1238.4 \\
AIC & 2861.8 & AIC & 1851.5 \\
z-value & 30.308 & $z$-value & 20.716 \\
$\rho$ & 0.79274 & Statistic & 0.69547 \\
Log Likelihood & 1434.918 & Log Likelihood & 1229.744 \\
Approximate Std. Error & 0.0081332 & Approximate Std. Error & 0.0081405 \\
\hline
\end{tabular}

\section{Discussion and Implications}

Using Predictive models of urban energy use driven by human mobility intrinsically account for the human activities of an urban population, which take place at certain times and locations [5,6]. However, our current knowledge about these patterns is based on today's population behavior and will inevitably vary under the influence of population growth and other changes in the future. Therefore, it is important to identify ways to dynamically project knowledge about urban population activities onto the relevant datasets and then use this to anticipate future energy demand. Opportunely, humans as sensors are the ideal dynamic sensors for such data collection.

Using 8,798,090 positional records gathered over the twelve (12) months of 2014 for the City of Chicago, we quantified returners populations' mobility [49] across 801 spatial divisions of the city. Using the historical monthly energy consumption over the same period, a spatial autocorrelation analysis revealed clustering patterns and positive spatial associations for returners' mobility and energy consumption across these areas. The temporal dependencies for returners' mobility paralleled with the temporal dependencies of energy consumption in terms of structural dependency, suggesting that the intensity of temporal fluctuations in the spatial variations of human mobility may well serve to explain the fluctuating intensities in energy use at different temporal dimensions. The findings of this study 
confirm the temporal manifestations of human mobility-driven building energy flux and support our proposed approach for the spatiotemporal prediction of energy use across urban spatial divisions. Taking into account the strength of spatial associations among and between human mobility and electricity consumption fluctuations by spatial division, we generated MAR and SAR models to predict electricity consumption in time and space in the City of Chicago. This study confirms previous research [56] on the relationship between human mobility and energy use across residential and commercial buildings in urban areas. However, this spatial association does not imply causality as human mobility and urban building energy consumption associations are complicated such that other underlying variables may be present causing the association. Our findings provide empirical evidence of an observed spatial correlation. Establishing causality from this evidence can and should be evaluated in future studies, taking into account the correlation strength and causality of various factors influencing on urban building energy consumption $[24,25,57]$.

This study represents an important step towards incorporating the space-time fluctuations of human mobility in urban populations to create better spatiotemporal predictions of energy demand at a high resolution in both time and space dimensions. Spatiotemporal predictions of energy use based on measures of human mobility are of paramount importance, since the continuing growth in both technology and urban populations [1] is anticipated to amplify the measure and variety of human activities, thus creating ever more complex built environments in our future cities, with even greater fluctuations in energy demands across time and space. Current urban scale energy predictive approaches [5-7,22,58-61] generally overlook the effects of consumer behaviors and thus will eventually fall short in terms of reliability. This study represents the first attempt to use the human mobility of an urban population as an indicator of their daily activities for the spatiotemporal predictions of energy demand in city neighborhoods. In order to cope with the continuing growth in population and the corresponding increase in urban activities, we need to develop a better understanding of the root causes of societally significant phenomena such as energy consumption. The spatiotemporal relationship between energy consumption and human mobility is a key element for creating and managing effective energy interventions and policies for urban areas. The level of space-time demand-side diversity will dictate corresponding energy resource allocation strategies and prepare our cities for a smarter future.

Given that half of those alive today now live in urban areas, rapid urbanization and the subsequent growth in energy consumption are important challenges that must be addressed. Segregating the spatiotemporal fluctuations in demand and supplying energy for citizens at times and locations that are needed is, at best, achieved through resource allocation via decentralized energy systems at different scales[62]. Understanding the spatiotemporal difference between supply and demand is a necessary step to determine distributed generation and becomes increasingly evident with renewable energy investment [22]. A greater awareness of how individuals move around urban open spaces and across the physical infrastructure of our urban environments (both communal and private) will enable us to build a more comprehensive understanding of how specific types of energy behavior are likely to be clustered in specific geographical spaces and temporal locations within urban areas. It will also enable us to identify the interdependencies between energy consumption, individual activities, and specific urban spatiotemporal features.

\section{Limitations}

This study is bound to possible limitations primarily as a result of data availability. First, the data collected from online social networks although representative, is not immune from varying tendencies of different segments of urban population and any results in this study are thus representative of this 
population. As yet, we lack appropriate data and analysis methods that will enable us to identify and use the full spectrum of spatiotemporal predictions. Our study is not immune from data availability constraints such as access to finer-grain temporal electricity consumption data. While our study does not suffer from spatial mismatches, this study inevitably had to deal with temporal mismatches (i.e., the electricity consumption data was from 2010, while the positional records accounting for human mobility were collected during 2014). The latest accessible, compatible Census Tract (2011) digital boundaries data was used for this study. Although 2010 has experienced comparably similar weather conditions, there has been a decline in temperature in winter 2014 compared to 2010, which may have impacted the results. However, since the primary source of space heating in the city of Chicago is gas, the effects on spatiotemporal distribution of electricity consumption are minimal. Future studies are encouraged to examine alternative datasets in order to characterize area-specific correlations across cities, using possibly finer spatiotemporal resolutions.

\section{Conclusions}

In this study we have demonstrated that there is a spatiotemporal dependency between human mobility and energy use in urban spaces. We have then presented a spatiotemporal predictive approach driven by human mobility of the urban population who dominates this dependency. Our findings suggest that human mobility can indeed account for the collective energy consumption in urban spaces, and further research should be considered to quantify and contextualize this relationship in predictive approaches across time and space. Overcoming the challenges of high population growth and the resulting increase in energy demand in urban areas requires a clear understanding the demand diversity and its association with the daily activities of the urban population at particular times and locations. Therefore, future research should seek to develop a better understanding of urban activity patterns across different functional locations using human mobility data and utilize the patterns identified to develop integrated predictive models that incorporate temporal elements of activity patterns and energy consumption. The capacity to identify spatial regions with similar temporal activities will enable us to assess their energy consumption and thus quantify the drivers of spatiotemporal fluctuations in energy use in urban areas and plan accordingly. This research is an important step towards achieving this goal.

\section{Acknowledgements}

This study was supported by the National Science Foundation under Grant No.1142379. Any opinions, findings, and conclusions or recommendations expressed in this material are those of the authors and do not necessarily reflect the views of the National Science Foundation.

\section{References}

[1] United Nations. World Urbanization Prospects: The 2014 Revision, Highlights (ST/ESA/SER.A/352). 2014. doi:10.4054/DemRes.2005.12.9.

[2] IEA. World Energy Outlook 2012. 2012.

[3] WorldBank. Cities and Climate Change. vol. 10. 2010. doi:10.1787/9789264091375-en.

[4] Agency IE. Transition to Sustainable Buildings. 2013. doi:10.1787/9789264202955-en.

[5] Fonseca JA, Schlueter A. Integrated model for characterization of spatiotemporal building energy consumption patterns in neighborhoods and city districts. Appl Energy 2015;142:247-65. doi:10.1016/j.apenergy.2014.12.068. 
[6] Mikkola J, Lund PD. Models for generating place and time dependent urban energy demand profiles. Appl Energy 2014;130:256-64. doi:10.1016/j.apenergy.2014.05.039.

[7] Zhu D, Tao S, Wang R, Shen H, Huang Y, Shen G, et al. Temporal and spatial trends of residential energy consumption and air pollutant emissions in China. Appl Energy 2013;106:17-24. doi:10.1016/j.apenergy.2013.01.040.

[8] Dean N. Residential energy demand: Consumption unzipped. Nat Energy 2016;1:16181. doi:10.1038/nenergy.2016.181.

[9] Pfenninger S, Hawkes A, Keirstead J. Energy systems modeling for twenty-first century energy challenges. Renew Sustain Energy Rev 2014;33:74-86. doi:10.1016/j.rser.2014.02.003.

[10] McGrath R, Pozdnoukhov A. A generative model of urban activities: simulating a population. ACM SIGKDD Int Work Urban Comput 2014.

[11] González MC, Hidalgo CA, Barabási A-L. Understanding individual human mobility patterns. Nature 2008;453:779-82. doi:10.1038/nature07850.

[12] Hasan S, Zhan X, Ukkusuri S V. Understanding urban human activity and mobility patterns using large-scale locationbased data from online social media. Proc 2nd ACM SIGKDD Int Work Urban Comput - UrbComp '13 2013:1. doi:10.1145/2505821.2505823.

[13] Widhalm P, Yang Y, Ulm M, Athavale S, González MC. Discovering urban activity patterns in cell phone data. Transportation (Amst) 2015;42:597-623. doi:10.1007/s11116-015-9598-x.

[14] Jain RK, Taylor JE, Peschiera G. Assessing eco-feedback interface usage and design to drive energy efficiency in buildings. Energy Build 2012;48:8-17. doi:10.1016/j.enbuild.2011.12.033.

[15] Chen J, Jain RK, Taylor JE. Block Configuration Modeling: A novel simulation model to emulate building occupant peer networks and their impact on building energy consumption. Appl Energy 2013;105:358-68.

doi:10.1016/j.apenergy.2012.12.036.

[16] The role of society in energy transitions. Nat Clim Chang 2016;6:539-539. doi:10.1038/nclimate3051.

[17] Howard B, Parshall L, Thompson J, Hammer S, Dickinson J, Modi V. Spatial distribution of urban building energy consumption by end use. Energy Build 2012;45:141-51. doi:10.1016/j.enbuild.2011.10.061.

[18] Boulaire F, Higgins A, Foliente G, McNamara C. Statistical modelling of district-level residential electricity use in NSW, Australia. Sustain Sci 2014;9:77-88. doi:10.1007/s11625-013-0206-8.

[19] Farzana S, Liu M, Baldwin A, Hossain MU. Multi-model prediction and simulation of residential building energy in urban areas of Chongqing, South West China. Energy Build 2014;81:161-9. doi:10.1016/j.enbuild.2014.06.007.

[20] Choudhary R. Energy analysis of the non-domestic building stock of Greater London. Build Environ 2012;51:243-54. doi:10.1016/j.buildenv.2011.10.006.

[21] Facchini A, Kennedy C, Stewart I, Mele R. The energy metabolism of megacities. Appl Energy 2017;186:86-95. doi:10.1016/j.apenergy.2016.09.025.

[22] Rauner S, Eichhorn M, Thran D. The spatial dimension of the power system: Investigating hot spots of Smart Renewable Power Provision. Appl Energy 2016;184:1038-50. doi:10.1016/j.apenergy.2016.07.031.

[23] Jain RK, Smith KM, Culligan PJ, Taylor JE. Forecasting energy consumption of multi-family residential buildings using support vector regression: Investigating the impact of temporal and spatial monitoring granularity on performance accuracy. Appl Energy 2014;123:168-78. doi:10.1016/j.apenergy.2014.02.057.

[24] Huebner GM, Hamilton I, Chalabi Z, Shipworth D, Oreszczyn T. Explaining domestic energy consumption - The comparative contribution of building factors, socio-demographics, behaviours and attitudes. Appl Energy 2015;159:589600. doi:10.1016/j.apenergy.2015.09.028.

[25] Huebner G, Shipworth D, Hamilton I, Chalabi Z, Oreszczyn T. Understanding electricity consumption: A comparative contribution of building factors, socio-demographics, appliances, behaviours and attitudes. Appl Energy 2016;177:692702. doi:10.1016/j.apenergy.2016.04.075.

[26] Zheng Y, Capra L, Wolfson O, Yang H. Urban Computing: Concepts, Methodologies, and Applications. ACM Trans Intell Syst Technol 2014;5:1-55. doi:10.1145/2629592.

[27] Becker R a., Cáceres R, Hanson K, Isaacman S, Loh JM, Martonosi M, et al. Human mobility characterization from 
cellular network data. Commun ACM 2013;56:74. doi:10.1145/2398356.2398375.

[28] Bagchi M, White PR. The potential of public transport smart card data. Transp Policy 2005;12:464-74. doi:10.1016/j.tranpol.2005.06.008.

[29] Zheng Y, Xie X. Location-based social networks: Locations. Comput with Spat Trajectories 2011:277-308. doi:10.1007/978-1-4614-1629-6_9.

[30] Zheng Y. Location-based social networks: Users. Comput with Spat Trajectories 2010:243-76. doi:http://dx.doi.org/10.1007/978-1-4614-1629-6_8.

[31] Hasan S, Schneider CM, Ukkusuri S V., González MC. Spatiotemporal Patterns of Urban Human Mobility. J Stat Phys 2013;151:304-18. doi:10.1007/s10955-012-0645-0.

[32] Yang Y, Chawla N V., Basu P, Prabhala P, La Porta T. Link prediction in human mobility networks. Proc 2013 IEEE/ACM Int Conf Adv Soc Networks Anal Mining, ASONAM 2013 2013:380-7. doi:10.1145/2492517.2492656.

[33] McInerney J, Stein S, Rogers A, Jennings NR. Breaking the habit: Measuring and predicting departures from routine in individual human mobility. Pervasive Mob Comput 2013;9:808-22. doi:10.1016/j.pmcj.2013.07.016.

[34] Lu X, Wetter E, Bharti N, Tatem AJ, Bengtsson L. Approaching the limit of predictability in human mobility. Nat Sci Reports 2013;3:2923. doi:10.1038/srep02923.

[35] Lu X, Bengtsson L, Holme P. Predictability of population displacement after the 2010 Haiti earthquake. Proc Natl Acad Sci U S A 2012;109:11576-81. doi:10.1073/pnas.1203882109.

[36] Zhao HX, Magoulès F. A review on the prediction of building energy consumption. Renew Sustain Energy Rev 2012;16:3586-92. doi:10.1016/j.rser.2012.02.049.

[37] Virote J, Neves-Silva R. Stochastic models for building energy prediction based on occupant behavior assessment. Energy Build 2012;53:183-93. doi:10.1016/j.enbuild.2012.06.001.

[38] Song C, Qu Z, Blumm N, Barabasi A-L. Limits of Predictability in Human Mobility. Science (80- ) 2010;327:1018-21. doi:10.1126/science.1177170.

[39] Yang N, Kong X, Wang F, Yu PS. When and Where: Predicting Human Movements Based on Social Spatial-Temporal Events. Sdm '14 2014:515-23. doi:10.1137/1.9781611973440.59.

[40] Alexander L, Jiang S, Murga M, González MC. Origin-destination trips by purpose and time of day inferred from mobile phone data. Transp Res Part C Emerg Technol 2015;58:240-50. doi:10.1016/j.trc.2015.02.018.

[41] Kitamura R, Chen C, Pendyala R a MM, Narayanan R. Micro-simulation of daily activity-travel patterns for travel. Transportation (Amst) 2000;27:25-51. doi:10.1023/A:1005259324588.

[42] Gambs S, Killijian M-O, Del Prado Cortez MN. Next place prediction using mobility Markov chains. Proc First Work Meas Priv Mobil MPM 2012 2012:1-6. doi:10.1145/2181196.2181199.

[43] Mraihi R, Harizi R, Mraihi T, Taoufik Bouzidi M. Urban air pollution and urban daily mobility in large Tunisia's cities. Renew Sustain Energy Rev 2015;43:315-20. doi:10.1016/j.rser.2014.11.022.

[44] Colizza V, Barrat A, Barthelemy M, Valleron AJ, Vespignani A. Modeling the worldwide spread of pandemic influenza: Baseline case and containment interventions. PLoS Med 2007;4:0095-110. doi:10.1371/journal.pmed.0040013.

[45] Balcan D, Gonçalves B, Hu H, Ramasco JJ, Colizza V, Vespignani A. Modeling the spatial spread of infectious diseases: The global epidemic and mobility computational model. J Comput Sci 2010;1:132-45. doi:10.1016/j.jocs.2010.07.002.

[46] Dias D, Tchepel O. Modelling of human exposure to air pollution in the urban environment: A GPS-based approach. Environ Sci Pollut Res 2014;21:3558-71. doi:10.1007/s11356-013-2277-6.

[47] Hanson S. Perspectives on the geographic stability and mobility of people in cities. Proc Natl Acad Sci U S A 2005;102:15301-6. doi:10.1073/pnas.0507309102.

[48] Shang J, Zheng Y, Tong W, Chang E, Yu Y. Inferring gas consumption and pollution emission of vehicles throughout a city. Proc 20th ACM SIGKDD Int Conf Knowl Discov Data Min - KDD ’14 2014:1027-36. doi:10.1145/2623330.2623653.

[49] Pappalardo L, Simini F, Rinzivillo S, Pedreschi D, Giannotti F, Barabási A-L. Returners and explorers dichotomy in human mobility. Nat Commun 2015;6:8166. doi:10.1038/ncomms9166. 
[50] Mohammadi N, Taylor JE. Urban Energy Flux : Human Mobility as a Predictor for Spatial Changes 2014.

[51] The City of Chicago Data Portal. City Chicago 2011. https://www.cityofchicago.org (accessed January 1, 2016).

[52] Fischer MM, Wang J. Spatial Data Analysis: Models, Methods and Techniques. 2011. doi:10.1007/978-3-642-21720-3.

[53] Moran PAP. Notes on Continuous Stochastic Phenomena. Biometrika 1950;37:17-23. doi:10.2307/2332142.

[54] Harrison L, Penny WD, Friston K. Multivariate autoregressive modeling of fMRI time series. Neuroimage 2003;19:147791. doi:10.1016/S1053-8119(03)00160-5.

[55] Whittle P. On Stationary Processes in the Plane. Biometrika 1954;41:434-49. doi:10.1093/biomet/41.3-4.434.

[56] Mohammadi N, Taylor JE, Wang Y. Towards Smarter Cities : Linking Human Mobility and Energy Use Fluctuations across Building Types. 50th Hawaii Int. Conf. Syst. Sci., 2017, p. 2824-33.

[57] IEA. World Energy Outlook. OECD/IEA,. Paris: International Energy Agency (IEA); 2016.

[58] Jin X, Mu Y, Jia H, Wu J, Xu X, Yu X. Optimal day-ahead scheduling of integrated urban energy systems. Appl Energy 2016;180:1-13. doi:10.1016/j.apenergy.2016.07.071.

[59] Ma J, Cheng JCP. Estimation of the building energy use intensity in the urban scale by integrating GIS and big data technology. Appl Energy 2016;183:182-92. doi:10.1016/j.apenergy.2016.08.079.

[60] Yin Y, Mizokami S, Aikawa K. Compact development and energy consumption: Scenario analysis of urban structures based on behavior simulation. Appl Energy 2015;159:449-57. doi:10.1016/j.apenergy.2015.09.005.

[61] Yeo I-A, Yoon S-H, Yee J-J. Development of an urban energy demand forecasting system to support environmentally friendly urban planning. Appl Energy 2013;110:304-17. doi:10.1016/j.apenergy.2013.04.065.

[62] Hiremath RB, Shikha S, Ravindranath NH. Decentralized energy planning; modeling and application-a review. Renew Sustain Energy Rev 2007;11:729-52. doi:10.1016/j.rser.2005.07.005. 


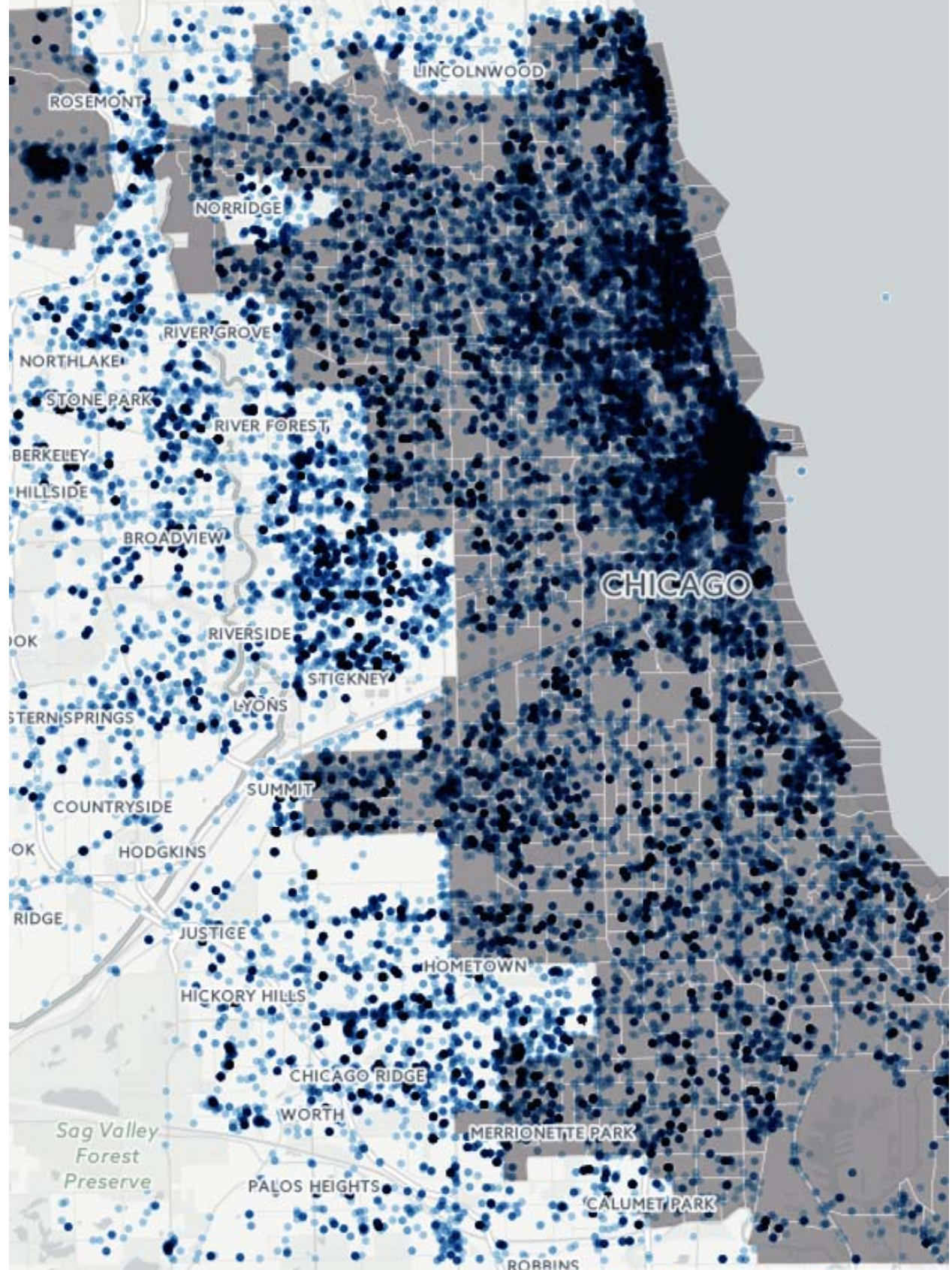




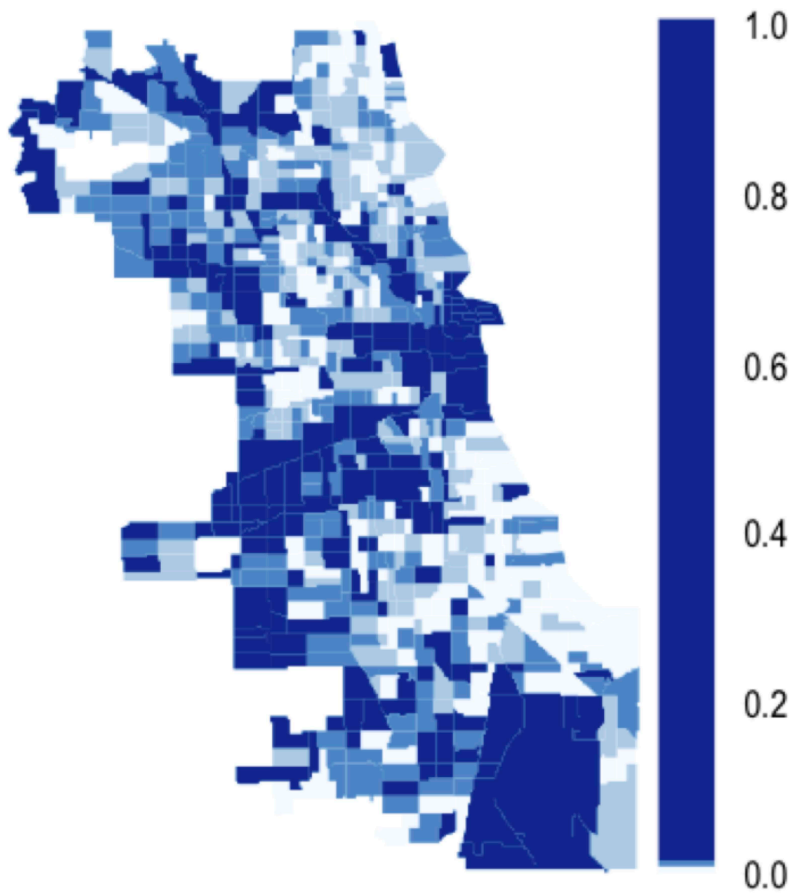

(a)

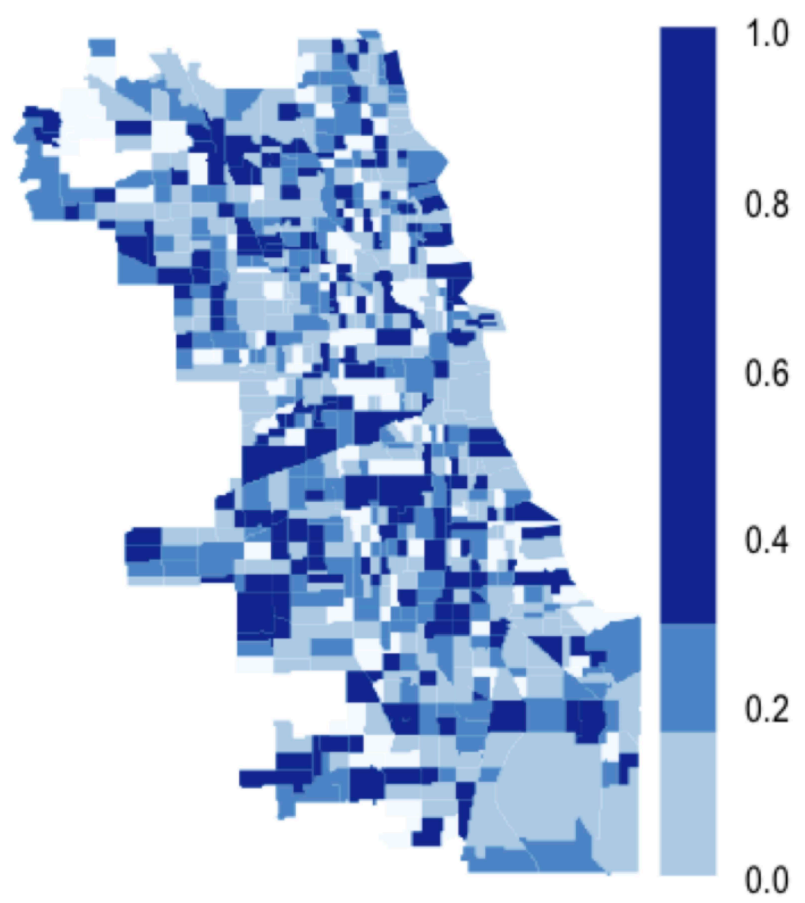

(b) 
Moran Scatterplot: Human Mobility: Returners

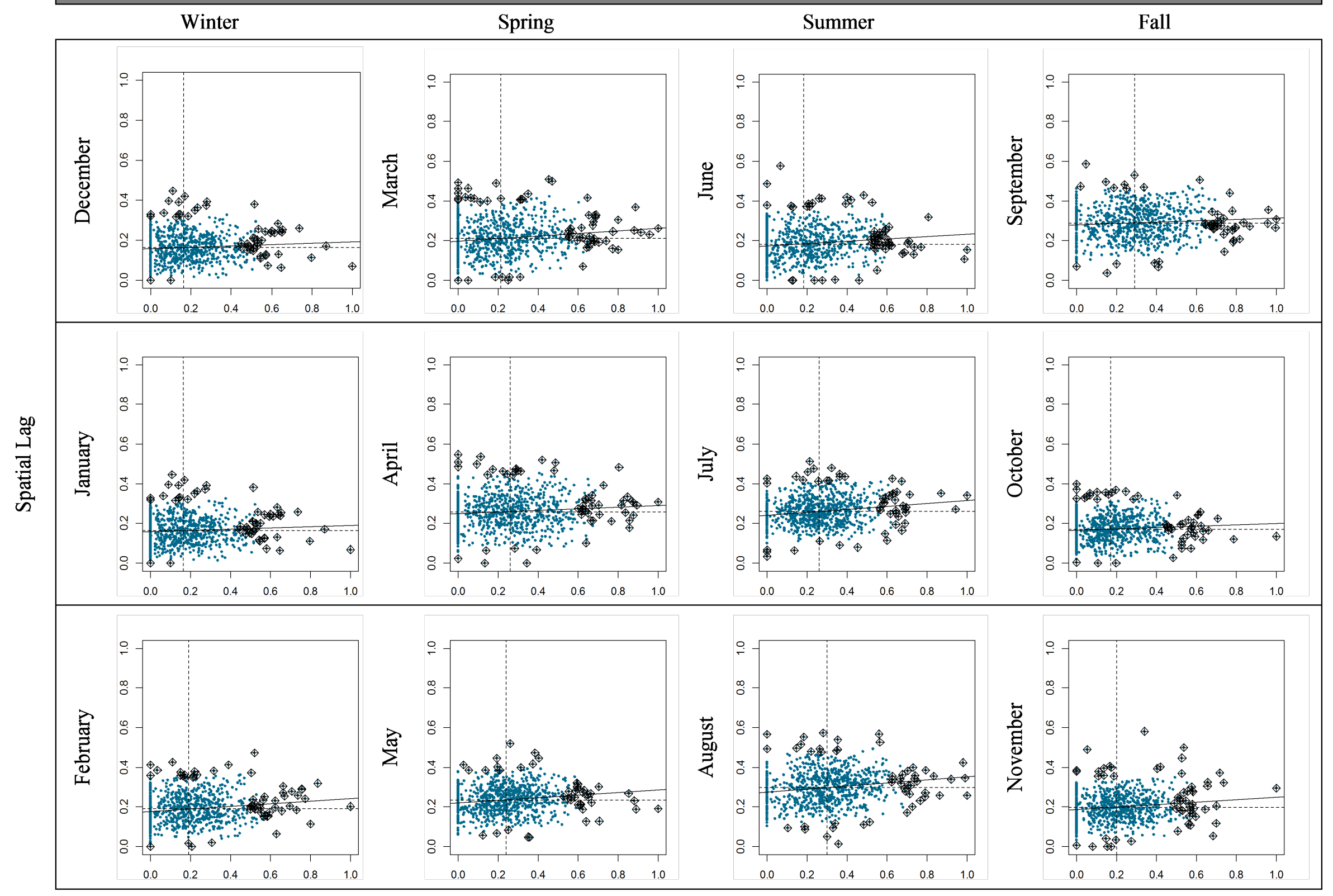

